\title{
Brownian motion in inhomogeneous suspensions
}

\author{
Mingcheng Yang ${ }^{*}$ and Marisol Ripoll ${ }^{\dagger}$ \\ Theoretical Soft-Matter and Biophysics, Institute of Complex Systems, Forschungszentrum Jülich, 52425 Jülich, Germany
}

(Received 8 November 2012; published 7 June 2013)

\begin{abstract}
The Langevin description of Brownian motion in inhomogeneous suspensions is here revisited. Inhomogeneous suspensions are characterized by a position-dependent friction coefficient, which can significantly influence the dynamics of the suspended particles. Outstanding examples are suspensions in confinement or in the presence of a temperature gradient. The Langevin approach in inhomogeneous systems encounters a fundamental difficulty related to the interpretation of the multiplicative noise induced by the position-dependent friction. We show that the so-called Ito-Stratonovich dilemma is originated by the violation of the macroscopic force balance condition in the traditional procedure of eliminating the fast variables. Repairing this deficit, we rederive the extended overdamped Langevin equation directly from the infradamped Langevin equation. This is without invoking the Fokker-Planck formalism, such that the self-completeness of the Langevin framework is restored. Furthermore, we derive the generalized forms of the drift-force relation and the Smoluchowski equation for inhomogeneous suspensions in a straightforward manner.
\end{abstract}

DOI: 10.1103/PhysRevE.87.062110

PACS number(s): 05.40.-a, 66.10.C-, 82.70.-y

\section{INTRODUCTION}

The physical essence of Brownian motion is substantial thermal fluctuations. It was first characterized as the random motion of pollen grains immersed in water [1]. Since then, the extension of this motion to explain the behaviors of a wide variety of systems has been enormous, including examples as diverse as soft matter systems or biological substances [2]. Most investigated systems have been traditionally assumed to be homogeneous, namely, with constant spatial properties. However, real systems of interest are often inhomogeneous, in which properties, such as the friction coefficient, vary with position. One relevant example is found in particles in confinement [3,4], where the diffusion coefficient of a particle decreases when approaching the wall due to hydrodynamic interactions. Another typical example is nonisothermal suspensions, in which inhomogeneity is induced by temperature gradients [5-8].

The inhomogeneity has a significant influence on the system dynamics. In contrast to homogeneous systems, the particle flux and drift velocity are related not only by the gradient of density but also by an additional contribution given by the gradient of the self-diffusion coefficient $[5,9,10]$. Similarly, the drift velocity in inhomogeneous systems has been shown not to uniquely depend on the driving force but to account for an additional contribution proportional to the gradient of mobility [9,11,12]. Recent experiments [13] have shown that disregarding the mobility gradient might even produce qualitatively wrong results when using the nonequilibrium force measurement method (NFM) [14-18], an advantaged force measurement technique where the force is extracted from particle drift. Therefore, to correctly consider the inhomogeneity effect is of critical importance for describing the system dynamics and for explaining experimental results.

\footnotetext{
*Present Address: Beijing National Laboratory for Condensed Matter Physics and Key Laboratory of Soft Matter Physics, Institute of Physics, Chinese Academy of Sciences, Beijing 100190, China; mcyang@iphy.ac.cn

${ }^{\dagger}$ m.ripoll@fz-juelich.de
}

Brownian motion studies have been performed following two alternative and equivalent approaches. The Langevin approach focuses on the particle trajectory while the FokkerPlanck approach investigates the particle distribution function [19-21]. These two descriptions of Brownian motion are first established in the so-called Fokker-Planck time scale $\tau_{\mathrm{FP}}$ [21]. This is a short time scale where the forceforce correlations decay and where the Brownian motion is described by the Langevin equation and the Fokker-Planck equation. Nevertheless, most relevant phenomena take place at longer times, on the so-called diffusive time scale $\tau_{\mathrm{D}}$, where the velocity-velocity correlations decay. On $\tau_{\mathrm{D}}$, the inertial terms of the Langevin equation and the velocity variable in the Fokker-Planck equation are unimportant, such that the adequate descriptions are the overdamped Langevin equation (OLE) and the Smoluchowski equation [19,21], as sketched in Fig. 1. For homogeneous systems, the elimination of the fast variables is independently implemented in both frameworks, and the resulting OLE and the Smoluchowski equation can be equivalently well applied. However, the Langevin route has a fundamental difficulty in inhomogeneous cases since the OLE obtained within the Langevin framework encounters the socalled Ito-Stratonovich dilemma [13,19,22-25]. Meanwhile, the Smoluchowski equation obtained from the Fokker-Planck equation is not affected. The difficulty is that the positiondependent friction in the OLE results in a multiplicative noise [19,26,27], and different interpretations of this noise lead to different theoretical predictions. This is a well-known problem that has been solved whether by disregarding the OLE in favor of the Smoluchowski equation $[5-8,12,19,28]$ or by reformulating the OLE based on the Smoluchowski equation $[11,29,30]$. This implies that the Langevin approach is not a self-complete scheme any longer, which relegates it to being a subordinate of the Fokker-Planck approach. Nonetheless, there is no reason to accept that a direct derivation of the correct OLE within the Langevin framework cannot be performed. Moreover, no physical reason has yet been revealed to explain the dilemma. In the most recent studies discussing the dilemma, their explanations are limited in inhomogeneous systems with an equilibrium steady state $[11,30]$. Therefore, 


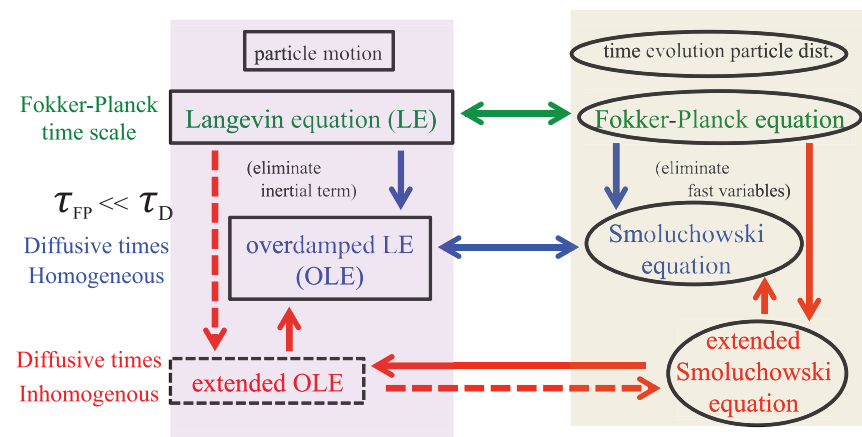

FIG. 1. (Color online) Schematic table of the theoretical approaches to study Brownian motion. Solid lines indicates equations and routes existing in the literature. Dashed lines mark the contributions of this work.

an independent derivation of the correct OLE in the Langevin framework without invoking the Smoluchowski equation is of theoretical interest and fundamental importance. In this paper, we provide such a derivation. We show that the procedure of eliminating fast variables in the Langevin framework cannot generally guarantee the macroscopic force balance condition, which constitutes the ultimate physical ground of the Ito-Stratonovich dilemma. By enforcing this condition, the correct extended OLE is directly obtained. This derivation is independent of the Smoluchowski equation, which restores the self-completeness of the Langevin approach. Our derivation is applicable to inhomogeneous systems both with and without an equilibrium steady state. From the obtained OLE, we furthermore derive a general drift-force relation and an extended Smoluchowski equation for inhomogeneous suspensions.

\section{LANGEVIN APPROACH AND MACROSCOPIC FORCE BALANCE}

The Langevin equation describes the time evolution in the Fokker-Planck time scale of a Brownian particle placed at position $x$ at time $t$ with velocity $\dot{x}$ as

$$
\ddot{x}=-\gamma(x) \dot{x}+F(x)+\chi(x) \eta(t) .
$$

Here, the particle mass has been set to unity; inhomogeneous conditions are considered in one dimension for simplicity. The particle acceleration is $\ddot{x}$, and $\gamma(x)$ is the friction coefficient. The total mechanical driving force $F(x)$ acts directly on the particle and includes externally applied forces, such as gravity, and the driving force exerted by the surrounding solvent, such as the thermophoretic force. The last term is the stochastic force with a position-dependent strength $\chi(x)$, and $\eta(t)$ is a Gaussian white noise with zero mean $\langle\eta(t)\rangle=0$ and variance $\left\langle\eta(t) \eta\left(t^{\prime}\right)\right\rangle=\delta\left(t-t^{\prime}\right)$. Because the noise $\eta(t)$ only causes a jump in velocity, $\eta(t)$ and $\chi(x)$ are statistically independent, i.e., $\langle\chi(x) \eta(t)\rangle=0$. Equation (1) is nothing but Newton's second law with a stochastic force.

The deterministic equation of motion of the particle can be obtained by performing the noise average of Eq. (1),

$$
\langle\ddot{x}\rangle=-\langle\gamma(x) \dot{x}\rangle+\langle F(x)\rangle .
$$

The term $-\langle\gamma(x) \dot{x}\rangle$ in Eq. (2) refers to the mean friction in the Fokker-Planck time scale. Thus, Eq. (2) can be interpreted as the deterministic force balance condition in the Fokker-Planck time scale, in which the driving force acting on the particle is averagely balanced by the corresponding friction and inertial forces. This just corresponds to Newton's second law with vanishing mean stochastic force.

In order to characterize the deterministic force balance equation in a more coarse-grained time scale $\Delta t \geqslant \tau_{\mathrm{FP}}$, Eq. (1) should be averaged not only over realizations of the noise but also over time. The force balance condition in Eq. (2) in the coarse-grained time scale is then

$$
\begin{aligned}
\frac{1}{\Delta t} \int_{t}^{t+\Delta t} d s\langle\ddot{x}\rangle= & -\frac{1}{\Delta t} \int_{t}^{t+\Delta t} d s\langle\gamma(x) \dot{x}\rangle \\
& +\frac{1}{\Delta t} \int_{t}^{t+\Delta t} d s\langle F(x)\rangle .
\end{aligned}
$$

The term on the left hand side refers to the mean inertial force $F_{i}$ in the time scale $\Delta t$. The first and second terms on the right hand side refer, respectively, to the mean friction force $F_{f}$ and the mean driving force $F_{d}$ in the time scale $\Delta t$. In the case when the diffusive time scale is considered $\left(\Delta t \geqslant \tau_{D}\right), F_{i}$ will vanish due to fast relaxation of the velocity variable [21]. Therefore, in the diffusive time scale the deterministic force balance equation becomes

$$
F_{f}(x)+F_{d}(x)=0
$$

which we will refer to as the macroscopic force balance condition. This equation is the most fundamental physical requirement that the equation of motion of a Brownian particle needs to satisfy in the diffusive time scale, and it will be later used to derive the correct OLE within the Langevin framework.

In the following, we explicitly calculate the mean friction and driving forces in the diffusive time $\left(\Delta t \geqslant \tau_{D}\right)$ in terms of measurable quantities as the mean displacement and the mean square displacement. The characterization of $F_{f}(x)$ in Eq. (4) requires the calculation of the following integral:

$$
\int_{t}^{t+\Delta t} d s \gamma[x(s)] \dot{x}(s)=\left.\gamma x\right|_{t} ^{t+\Delta t}-\int_{t}^{t+\Delta t} d s \dot{\gamma} x .
$$

For notational convenience and clarity, note that the argument of the variables is not always specified. The integral has been calculated by parts, and a slow variation of $\gamma(x)$ with $x$ now needs to be considered. The friction function can then be approximated by a Taylor expansion around $x(t)$, as $\gamma[x(t+\Delta t)] \simeq \gamma[x(t)]+\gamma^{\prime}[x(t)] \Delta x+\frac{1}{2} \gamma^{\prime \prime}[x(t)] \Delta x^{2}$, with $\Delta x=x(t+\Delta t)-x(t)$ and where the primes denote spatial derivatives, $\gamma^{\prime} \equiv \partial \gamma / \partial x$. The first term on the right hand side of Eq. (5) is then

$$
\begin{aligned}
\left.\gamma x\right|_{t} ^{t+\Delta t}= & \left\{\gamma^{\prime}[x(t)] x(t)+\gamma[x(t)]\right\} \Delta x \\
& +\left\{\gamma^{\prime}[x(t)]+\frac{1}{2} \gamma^{\prime \prime}[x(t)] x(t)\right\} \Delta x^{2},
\end{aligned}
$$

where higher order terms are neglected. The second term on the right hand side of Eq. (5) can be evaluated by performing the 
Taylor expansion $\gamma^{\prime}[x(s)] \simeq \gamma^{\prime}[x(t)]+\gamma^{\prime \prime}[x(t)]\{x(s)-x(t)\}$ as

$$
\begin{aligned}
\int_{t}^{t+\Delta t} d s \dot{\gamma} x & =\int_{t}^{t+\Delta t} d s \gamma^{\prime}[x(s)] x(s) \dot{x}(s) \\
& =\gamma^{\prime} x(t) \Delta x+\frac{1}{2}\left\{\gamma^{\prime}+\gamma^{\prime \prime} x(t)\right\} \Delta x^{2} .
\end{aligned}
$$

Inserting Eqs. (6) and (7) in Eq. (5), we have

$$
F_{f}(x)=-\frac{1}{\Delta t}\left(\gamma\langle\Delta x\rangle+\frac{1}{2} \gamma^{\prime}\left\langle\Delta x^{2}\right\rangle\right) .
$$

The mean driving force in Eq. (4) can be directly calculated by Taylor expanding $F[x(s)]$ around $x(t)$,

$$
\begin{aligned}
F_{d}(x) & =\frac{1}{\Delta t} \int_{t}^{t+\Delta t} d s\left\langle F[x(t)]+F^{\prime}[x(t)]\{x(s)-x(t)\}\right\rangle \\
& =F(x)+\frac{F^{\prime}(x)}{\Delta t} \int_{t}^{t+\Delta t} d s\langle x(s)-x(t)\rangle \simeq F(x),
\end{aligned}
$$

where the term neglected in the last approximation can be shown to be proportional to $F^{\prime}(x)\langle\Delta x\rangle$, which provides a higher order contribution.

\section{STANDARD OVERDAMPED LANGEVIN EQUATION}

In the diffusive time scale, it is well known that the infradamped LE in Eq. (1) can be significantly simplified. The inertial term in Eq. (1) can be neglected [21] in the time scale $\tau_{\mathrm{D}}$, such that the standard accepted procedure to eliminate the fast variables naively simplifies Eq. (1) to the following overdamped LE:

$$
\dot{x}=\mu(x) F(x)+g(x) \eta(t),
$$

with $\mu=1 / \gamma$ being the mobility of the particle and $g=\chi / \gamma$. Note that, here, all the variables should be understood in the diffusive time scale, in contrast to those in Eq. (1), which are to be understood in the Fokker-Planck time scale. This implies that all related quantities like the velocities or friction are not necessarily the same at both time scales since variables in the diffusive scale are the time average of the variables on the Fokker-Planck scale, as discussed in Sec. II. In homogeneous systems, Eq. (10) provides a correct description of the dynamics of Brownian particles, and it is therefore frequently employed. However, Eq. (10) is generally invalid in the case of inhomogeneous systems. This is due to the fact that now the noise $\eta(t)$ causes a jump in the position of the particle rather than in its velocity. It is then important to consider when the noise strength $g(x)$ needs to be evaluated, whether before the jump, after the jump, or at any other intermediate point. The standard procedure to account for all possible evaluations of the noise is to parametrize the related functions in terms of a continuous variable $\alpha \in[0,1]$. The noise amplitude can be then evaluated as $g[x(t)+\alpha \Delta x]$, with $\Delta x$ being the jump in position. When $\alpha=0$, the noise is evaluated before the jump, corresponding to the Ito convention, and when $\alpha=1 / 2$, the noise is evaluated just in between two consecutive jumps, corresponding to the Stratonovich convention $[11,19]$. Different choices or conventions for the noise evaluation employed in Eq. (10) may lead to different results, which is known as the Ito-Stratonovich dilemma [19].
The existence of such a dilemma contradicts the basic principle that physical results are independent of mathematical treatments since real systems have unique thermodynamics and dynamics. Although the dilemma has been solved with different approaches, no physical reason for its existence has yet been found.

The fundamental problem is that the OLE in Eq. (10) does not satisfy the macroscopic force balance condition in the diffusive time scale, Eq. (4). In order to see this, we simply average Eq. (10) over the noise,

$$
\langle\gamma(x) \dot{x}\rangle=\langle F(x)\rangle+\langle\chi(x) \eta(t)\rangle .
$$

We now separately discuss each term. As already mentioned, $\chi(x)$ depends on $\eta(t)$ through $\alpha \Delta x$, such that the stochastic term $\langle\chi(x) \eta(t)\rangle$ is, in general, nonvanishing, contradicting the character of the stochastic force. This suggests that a term compensating the stochastic force is missing when the traditional elimination procedure is applied to inhomogeneous systems. Averaging the driving force by considering all possible noise conventions and neglecting higher order terms similarly to Eq. (9), $\langle F[x(t)+\alpha \Delta x]\rangle \simeq F[x(t)]$, show that $\langle F(x)\rangle$ is just the mean driving force $F_{d}$. Finally, the term $-\langle\gamma(x) \dot{x}\rangle$ is generally not equal to the mean friction in the diffusion time scale $F_{f}$, unless $\gamma(x)$ is constant. This becomes particularly clear when considering all possible noise conventions to calculate this term,

$$
\begin{aligned}
-\langle\gamma(x) \dot{x}\rangle & =-\left\langle\gamma[x(t)+\alpha \Delta x] \frac{\Delta x}{\Delta t}\right\rangle \\
& =-\frac{1}{\Delta t}\left(\gamma\langle\Delta x\rangle+\alpha \gamma^{\prime}\left\langle\Delta x^{2}\right\rangle\right) .
\end{aligned}
$$

Comparing with Eq. (8), it can be seen that it is only in the particular case of $\alpha=1 / 2$ that $-\langle\gamma(x) \dot{x}\rangle$ is equal to $F_{f}$. This implies that an additional term compensating the friction force is missing from the traditional elimination procedure. Note that even the combination of $-\langle\gamma(x) \dot{x}\rangle+\langle\chi(x) \eta(t)\rangle$ still does not correspond to the mean friction force $F_{f}$. Therefore, it is here proved that Eq. (10) does not obey the macroscopic force balance condition in Eq. (4) for inhomogeneous systems. It is important to emphasize that the OLE is the particle equation of motion, which should be the result of the balance of the relevant forces. So the traditional elimination procedure and the OLE in Eq. (10) need to be revised.

\section{EXTENDED OVERDAMPED LANGEVIN EQUATION}

To establish the correct OLE, which satisfies the macroscopic force balance in Eq. (4), the friction and stochastic forces need to be properly coarse grained, in contrast to Eq. (10). In a similar way to Ref. [11], we consider an extended OLE with an additional and yet unknown contribution $f_{1}(x)$ which includes all possible missing terms,

$$
\dot{x}(t)=\mu(x) F(x)+f_{1}(x)+g(x) \eta(t) .
$$

For the sake of convenience, we define the global drift term,

$$
\mathscr{F}(x)=\mu(x) F(x)+f_{1}(x) .
$$

In order to apply the macroscopic force balance condition, we need to quantify the mean displacement $\langle\Delta x\rangle$ and the mean 
square displacement $\left\langle\Delta x^{2}\right\rangle$. We first integrate Eq. (13),

$$
\Delta x=\int_{t}^{t+\Delta t} d s\{\mathscr{F}[x(s)]+g[x(s)] \eta(s)\} .
$$

The calculation of this integral requires us to assume that $\mu(x)$, $F(x), f_{1}(x)$, and $g(x)$ only vary slowly with $x$. To account for all possible noise conventions, Eq. (15) can be generally written in terms of the variable $\alpha \in[0,1]$,

$$
\Delta x=\mathscr{F}[x(t)+\alpha \Delta x] \Delta t+g[x(t)+\alpha \Delta x] \int_{t}^{t+\Delta t} d s \eta(s) .
$$

A Taylor expansion to first order in $\Delta t$ can now be performed,

$$
\begin{aligned}
\Delta x= & \mathscr{F}[x(t)] \Delta t+g[x(t)] \int_{t}^{t+\Delta t} d s \eta(s) \\
& +g^{\prime}[x(t)] \alpha \Delta x \int_{t}^{t+\Delta t} d s \eta(s) .
\end{aligned}
$$

Thus, Eq. (17) provides an iterative procedure that allows us to estimate $\Delta x$ as

$$
\begin{aligned}
\Delta x= & \mathscr{F} \Delta t+g \int_{t}^{t+\Delta t} d s \eta(s) \\
& +\alpha g^{\prime} g \int_{t}^{t+\Delta t} d s \int_{t}^{t+\Delta t} d \tilde{s} \eta(s) \eta(\tilde{s}),
\end{aligned}
$$

where higher-order terms have been ignored. Calculating the noise average, we obtain the mean particle displacement

$$
\langle\Delta x\rangle=\mathscr{F} \Delta t+\alpha g g^{\prime} \Delta t .
$$

This equation indicates that the measurable drift velocity of a single particle, $v_{d}=\langle\Delta x\rangle / \Delta t$, may depend on the choice of $\alpha$ (an example of the Ito-Stratonovich dilemma), unless this dependence is canceled by the functional dependence of the additional $f_{1}(x)$. The mean square displacement can also be calculated as

$$
\left\langle\Delta x^{2}\right\rangle=g^{2}(x) \Delta t .
$$

The OLE considers the system to be in the diffusion time scale, such that the mean square displacement can be related to the local self-diffusion coefficient of the particle and $g^{2}(x)=$ $2 D(x)$.

With the averaged values in Eqs. (19) and (20), the mean friction force in the diffusive time scale $F_{f}$ is calculated in terms of Eq. (8) as

$$
F_{f}(x)=-\gamma(x) \mathscr{F}(x)-\alpha \gamma(x) D^{\prime}(x)-\gamma^{\prime}(x) D(x) .
$$

The consideration of the macroscopic force balance condition of Eq. (4) with Eqs. (21) and (9) results in

$$
f_{1}(x)=\frac{D(x)}{\mu(x)} \mu^{\prime}(x)-\alpha D^{\prime}(x) .
$$

Thus, the missed terms in the traditional elimination procedure are recovered. Substituting Eq. (22) into Eq. (13), the correct extended OLE reads then

$$
\dot{x}=\mu(x) F(x)+\frac{D(x)}{\mu(x)} \mu^{\prime}(x)-\alpha D^{\prime}(x)+g(x) \eta(t) .
$$

Now, the extended OLE satisfies the fundamental physical requirement of Eq. (4). Note that the last two terms on the right side of Eq. (23) have a vanishing noise average, namely, $\left\langle-\alpha D^{\prime}(x)+g(x) \eta(t)\right\rangle=0$, which can be shown just by Taylor expanding $g(x)$ around $x(t)$ and then using Eq. (18). This follows the property of the stochastic force. Meanwhile $\left\langle-\dot{x}+\frac{D(x)}{\mu(x)} \mu^{\prime}(x)\right\rangle$ can be understood as the frictional contribution of $F_{f} / \gamma$ in terms of Eq. (8). In this sense, the two additional terms are not new additional forces but originate from the friction and the stochastic forces when properly coarse graining the time from the Fokker-Planck into the diffusive time scale. In other words, the existence of $f_{1}(x)$ indicates that the friction and the stochastic force generally have different forms for the LE in Eq. (1) and the OLE in Eq. (23). It is therefore of fundamental importance to distinguish whether the system under study is being investigated in the Fokker-Planck or the diffusive time scale.

Apart from homogeneous systems, there is one case in which the two additional terms in Eq. (23) cancel each other. This is in the case of an isothermal suspension where the Einstein relation holds, $D(x)=k_{B} T \mu(x)$, in addition to the special choice of $\alpha=1$. This is the reason why experimental results of the drift velocity of colloidal particles in recent studies $[13,22]$ could be satisfactorily explained by employing the standard OLE in Eq. (10) together with the special convention $\alpha=1$, which was then not otherwise justified. Although Eq. (23) explicitly depends on the convention of the noise evaluation, in the next sections we show that the averaged physical quantities no longer depend on the noise convection.

The extended OLE in Eq. (23) constitutes a slight generalization of existing versions of the same equation. For the case of the Stratonovich convention $(\alpha=1 / 2)$, Eq. (23) was already obtained three decades ago [29], although through the Smoluchowski equation. More recently, Lau and Lubensky [11] also considered the existence of additional terms in the OLE. In order to determine such terms, they first obtain the Smoluchowski equation and then impose the condition that the system needs to evolve to the Boltzmann equilibrium distribution at long times. Their results are therefore valid in systems with a well-defined equilibrium state; however, systems with intrinsic nonequilibrium constraints are excluded. This is the case of nonisothermal systems. This is not a restriction in our case since the force balance condition is still satisfied far from equilibrium. Additionally, our expression in Eq. (23) trivially reduces to that of Ref. [11] when assuming the Einstein relation together with the isothermal condition [31].

The overdamped Langevin equation is therefore established based simply on the force balance condition, such that additional considerations are necessary to ensure equilibrium or irreversible thermodynamics. In the linear response regime, the fluctuation dissipation relation [19-21] should be satisfied, which enforces a particular relation between the frictional coefficient and the self-diffusion coefficient. Moreover, if the driving force is not externally applied, such as gravity, but arises from nonequilibrium effects, like the thermophoretic force, then the Onsager's force-flux type linear relation in the irreversible thermodynamics needs to be used to specify this driving force [32]. If the system is far away from the equilibrium state, the validity of a general fluctuation-dissipation relation is then questioned. The frictional and diffusion coefficients have to be specified as those of the system under study. They can be determined from microscopic theories, 
experiments, or simulations. Furthermore, the extended OLE could be regarded as a starting point to extend the recently developed "stochastic thermodynamics theory" [33-36] to inhomogeneous systems.

\section{DRIFT VELOCITY}

Of special relevance is the drift velocity of a Brownian particle, which can be precisely quantified. The theoretical prediction can be directly obtained by combining Eqs. (19) and (22),

$$
v_{d}=\mu(x) F(x)+\frac{D(x)}{\mu(x)} \mu^{\prime}(x) .
$$

This means that the presence of a systematic inhomogeneity together with the only requirement of macroscopic force balance translates into an additional contribution to the particle drift velocity. Naturally, this contribution does not depend on the performed choice for the noise interpretation, only on the spatial dependence of the diffusion coefficient and the mobility.

Single-particle tracking techniques can now experimentally measure the mean displacement and the mean square displacement of the Brownian particle [13]. Thus, the drift velocity and the diffusion coefficient of the particle can be locally quantified, which allows us to extract the mechanical driven force and other transport coefficients with Eq. (24). Furthermore, recent experimental techniques [37] permit us to explore the inertial regime of the Brownian motion in some particular systems and hence to determine the instantaneous velocity of the particle. This provides a direct way to obtain the mechanical driven force, such that the prediction in Eq. (24) could be tested by means of experiments in addition to standard computer simulations. On the other hand, the measurements over short times can also provide valuable information about behavior in the crossover between the Fokker-Planck and the diffusive regimes.

Our theoretical derivation has so far not assumed any particular relation between the diffusion coefficient and the mobility, so Eqs. (23) and (24) are general within the validity of the performed approximations. If the system is far beyond equilibrium, the definition $D(x) / \mu(x)=k_{B} T_{\mathrm{eff}}(x)$ has been commonly employed [38-40], where $T_{\text {eff }}(x)$ could be understood as an effective temperature. When the system is in the linear response regime, the local equilibrium approximation is valid, and the Einstein relation $D(x)=k_{B} T(x) \mu(x)$ is obeyed, with $T(x)$ being the local temperature. In such a case, the drift velocity becomes

$$
v_{d}=\mu(x) F(x)+k_{B} T(x) \mu^{\prime}(x) .
$$

This is a known expression that has previously been inferred from the van Kampen equation [9,12] and that here we obtain directly from the extended OLE. The validity of this equation has already been validated by means of computer simulations for a Brownian particle and for a binary mixture of LennardJones particles in the presence of a temperature gradient [9].

For inhomogeneous systems with a uniform temperature that follow the Einstein relation, the drift velocity can be written as

$$
v_{d}=\mu(x) F(x)+D^{\prime}(x) .
$$

This expression has been previously obtained [11,30] and has also been quantitatively verified in two different experimental setups [13,22].

The additional drift term produced by the inhomogeneous character of the system can not only produce certain mismatch between the driving force and the related drift velocity, but it can even induce a sign change between them $[9,13]$, such that its consideration is of high importance when techniques such as single-particle tracking or NFM are to be employed.

\section{THE EXTENDED SMOLUCHOWSKI EQUATION}

As already stated and summarized in Fig. 1, alternatively to the particle equation of motion provided by the Langevin equation, the Brownian motion can be described by the Smoluchowski equation, which considers the time evolution of the particle distribution function $P(x, t)$. This evolution can be obtained from the relation of the probability densities at two different times $[19,41,42]$,

$$
P(x, t+\Delta t)=\int d x_{0} W\left(x, t+\Delta t \mid x_{0}, t\right) P\left(x_{0}, t\right),
$$

where the transition probability between both states can be expressed and Taylor expanded as

$$
\begin{aligned}
W\left(x, t+\Delta t \mid x_{0}, t\right) & =\langle\delta[x-x(t+\Delta t)]\rangle_{x_{0}, t} \\
& =\left[1-\langle\Delta x\rangle \frac{\partial}{\partial x}+\frac{1}{2}\left\langle\Delta x^{2}\right\rangle \frac{\partial^{2}}{\partial x^{2}}\right] \delta\left(x-x_{0}\right)
\end{aligned}
$$

and where higher order terms have been ignored. Thus, inserting Eq. (28) in Eq. (27), we obtain

$$
\frac{\partial P(x, t)}{\partial t}=-\frac{\partial}{\partial x}\left[\frac{\langle\Delta x\rangle}{\Delta t}-\frac{1}{2} \frac{\partial}{\partial x} \frac{\left\langle\Delta x^{2}\right\rangle}{\Delta t}\right] P(x, t) .
$$

Equations (27)-(29) constitute the standard derivation of the Smoluchowski equation from the OLE, and now the values for the average displacement and mean square displacement obtained from the extended OLE in Eqs. (24) and (20) can be employed, yielding

$$
\frac{\partial P(x, t)}{\partial t}=-\frac{\partial}{\partial x}\left[\mu(x) F(x)-\mu(x) \frac{\partial}{\partial x} \frac{D(x)}{\mu(x)}\right] P(x, t) .
$$

Here it can be seen that there is no remaining dependence on the noise interpretation since the observables $\langle\Delta x\rangle$ and $\left\langle\Delta x^{2}\right\rangle$ are already not convection dependent. Equation (30) was originally derived by Sancho et al. [29] through an involved adiabatic elimination procedure. The corresponding particle flux is

$$
J(x, t)=\mu(x) F(x) P(x, t)-\mu(x) \frac{\partial}{\partial x}\left[\frac{D(x)}{\mu(x)} P(x, t)\right] .
$$

When the Einstein relation is valid, Eq. (31) reduces to the van Kampen flux formula [5,43],

$J(x, t)=\mu(x) F(x) P(x, t)-\mu(x) \frac{\partial}{\partial x}\left[k_{B} T(x) P(x, t)\right]$.

Finally, we want to emphasize that Eq. (32) provides a very convenient framework to study nonisothermal suspensions [43-46]. The transport of mass due to the temperature 
gradient is known as thermodiffusion [47-49]. This phenomenon has been shown to be of relevance in numerous fields like separation of macromolecules [50] and microfluidic applications [51-53]. In this case, the mechanical driving force $F(x)$ should be understood as the thermophoretic force exerted on suspended particles by the inhomogeneous environment induced by the temperature gradient. This thermophoretic force can now be experimentally determined with techniques such as single-particle tracking together with the drift velocity expression in Eq. (25) [9,51]. Based on Eq. (32), we have recently proposed [10] a new expression to characterize the main thermodiffusion transport properties like the Soret coefficient, which has been quantitatively verified by means of computer simulations. Similarly, we expect that Eqs. (24) and (31) will also constitute a valuable tool in the investigation of various inhomogeneous systems far from equilibrium.

\section{CONCLUSIONS}

In the present work, we revisit the Langevin description of Brownian motion in inhomogeneous suspensions. We show that the Ito-Stratonovich dilemma is originated by the violation of the macroscopic force balance condition in the traditional procedure of eliminating the fast variables. This violation contradicts in its grounds a proper particle equation of motion. By requiring the macroscopic force balance condition, we rederive the extended OLE directly from the infradamped Langevin equation, which is therefore independent of the Fokker-Planck and Smoluchowski equations. This procedure restores the self-completeness of the Langevin approach when applied to inhomogeneous suspensions. This provides a rigorous physical foundation for the overdamped approximation and a direct way to elegantly solve the Ito-Stratonovich dilemma. Our results are applicable to inhomogeneous systems with and without an equilibrium steady state, like nonisothermal suspensions. Furthermore, starting with the extended OLE, we obtain the generalized drift-force relation and the extended Smoluchowski equation for the inhomogeneous Brownian motion. Although the most general case has not yet been verified, there is diverse evidence to prove the drift-force relation and the mass flux equation when the Einstein relation is fulfilled; this is the van Kampen equation. The contributions presented in this article constitute an important, and until now missing, piece of information that completes the existing picture of Brownian motion in inhomogeneous systems.

\section{ACKNOWLEDGMENTS}

We would like to thank A. Würger for pointing out Ref. [11] to us and H. R. Ma for comments on our manuscript.
[1] R. Brown, Philos. Mag. 4, 161 (1828).

[2] E. Frey and K. Kroy, Ann. Phys. (NY) 14, 20 (2005).

[3] H. Brenner, Chem. Eng. Sci. 16, 242 (1961).

[4] P. Holmqvist, J. K. G. Dhont, and P. R. Lang, Phys. Rev. E 74, 021402 (2006).

[5] N. van Kampen, J. Phys. Chem. Solids 49, 673 (1988).

[6] A. Pérez-Madrid, J. Rubi, and P. Mazur, Phys. A 212, 231 (1994).

[7] M. Matsuo and S. Sasa, Phys. A 276, 188 (2000).

[8] C. Lopez and U. Marini Bettolo Marconi, Phys. Rev. E 75, 021101 (2007).

[9] M. Yang and M. Ripoll, J. Chem. Phys. 136, 204508 (2012).

[10] M. Yang and M. Ripoll, J. Phys. Condens. Matter 24, 195101 (2012).

[11] A. W. C. Lau and T. C. Lubensky, Phys. Rev. E 76, 011123 (2007).

[12] E. Bringuier and A. Bourdon, J. Non-Equilib. Thermodyn. 32, 221 (2007).

[13] G. Volpe, L. Helden, T. Brettschneider, J. Wehr, and C. Bechinger, Phys. Rev. Lett. 104, 170602 (2010).

[14] P. Poulin, V. Cabuil, and D. A. Weitz, Phys. Rev. Lett. 79, 4862 (1997).

[15] J. C. Neto, R. Dickman, and O. N. Mesquita, Phys. A 345, 173 (2005).

[16] S. K. Sainis, V. Germain, and E. R. Dufresne, Phys. Rev. Lett. 99, 018303 (2007).

[17] P. Wu, R. Huang, C. Tischer, A. Jonas, and E.-L. Florin, Phys. Rev. Lett. 103, 108101 (2009).

[18] J. W. Merrill, S. K. Sainis, and E. R. Dufresne, Phys. Rev. Lett. 103, 138301 (2009).
[19] N. van Kampen, Stochastic Processes in Physics and Chemistry (North Holland, Amsterdam, 1992).

[20] P. M. Chaikin and T. C. Lubensky, Principles of Condensed Matter Physics (Cambridge University Press, New York, 1995).

[21] J. K. G. Dhont, An Introduction to Dynamics of Colloids (Elsevier, Amsterdam, 1996).

[22] P. Lançon, G. Batrouni, L. Lobry, and N. Ostrowsky, Europhys. Lett. 54, 28 (2001).

[23] P. Lançon, G. Batrouni, L. Lobry, and N. Ostrowsky, Phys. A 304, 65 (2002).

[24] R. Mannella and P. V. E. McClintock, Phys. Rev. Lett. 107, 078901 (2011).

[25] G. Volpe, L. Helden, T. Brettschneider, J. Wehr, and C. Bechinger, Phys. Rev. Lett. 107, 078902 (2011).

[26] P. Arnold, Phys. Rev. E 61, 6091 (2000).

[27] D. C. Morse, Adv. Chem. Phys. 128, 65 (2004).

[28] A. M. Jayannavar and M. C. Mahato, Pramana 45, 369 (1995).

[29] J. M. Sancho, M. Miguel, and D. Dürr, J. Stat. Phys. 28, 291 (1982).

[30] J. M. Sancho, Phys. Rev. E 84, 062102 (2011).

[31] Note that there is a typo (an additional factor of 2) in the second equality of Eq. (2.21) of Ref. [11].

[32] S. R. de Groot and P. Mazur, Nonequilibrium Thermodynamics (Dover, New York, 1984).

[33] U. Seifert, Rep. Prog. Phys. 75, 126001 (2012).

[34] T. Hatano and S. I. Sasa, Phys. Rev. Lett. 86, 3463 (2001).

[35] U. Seifert, Phys. Rev. Lett. 95, 040602 (2005).

[36] T. Speck and U. Seifert, Europhys. Lett. 74, 391 (2006).

[37] T. Li, S. Kheifets, D. Medellin, and M. Raizen, Science 328, 1673 (2010). 
[38] L. Berthier and J. L. Barrat, Phys. Rev. Lett. 89, 095702 (2002).

[39] D. Rings, R. Schachoff, M. Selmke, F. Cichos, and K. Kroy, Phys. Rev. Lett. 105, 090604 (2010).

[40] L. Joly, S. Merabia, and J. L. Barrat, Europhys. Lett. 94, 50007 (2011).

[41] H. Risken, The Fokker Planck Equation (Springer, Berlin, 1989).

[42] R. K. Pathria, Statistical Mechanics (Butterworth-Heinemann, Oxford, 1996).

[43] M. E. Widder and U. M. Titulaer, Phys. A 154, 452 (1989).

[44] E. Bringuier and A. Bourdon, Phys. Rev. E 67, 011404 (2003).

[45] J. K. G. Dhont, J. Chem. Phys. 120, 1632 (2004).
[46] S. Fayolle, T. Bickel, S. Le Boiteux, and A. Würger, Phys. Rev. Lett. 95, 208301 (2005).

[47] S. Wiegand, J. Phys. Condens. Matter 16, R357 (2004).

[48] C. Debuschewitz and W. Köhler, Phys. Rev. Lett. 87, 055901 (2001).

[49] R. Piazza and A. Parola, J. Phys. Condens. Matter 20, 153102 (2008).

[50] J. Giddings, Science 260, 1456 (1993).

[51] S. Duhr and D. Braun, Proc. Natl. Acad. Sci. USA 103, 19678 (2006).

[52] H.-R. Jiang, H. Wada, N. Yoshinaga, and M. Sano, Phys. Rev. Lett. 102, 208301 (2009).

[53] M. Yang and M. Ripoll, Phys. Rev. E 84, 061401 (2011). 